\title{
Activity of human AP-endonuclease APE1 on DNA- and RNA-substrates forming non-canonical structures
}

\author{
Anastasiia T. Davletgildeeva \\ Institute of Chemical Biology and Fundamental Medicine \\ Siberian Branch of Russian Academy of Sciences \\ Novosibirsk, Russia \\ davleta94@gmail.com \\ Olga S. Fedorova \\ Institute of Chemical Biology and Fundamental Medicine \\ Siberian Branch of Russian Academy of Sciences \\ Novosibirsk, Russia \\ fedorova@niboch.nsc.ru
}

\begin{abstract}
Human apurinic/apyrimidinic endonuclease APE1 is responsible for detecting and initiating the elimination of AP-sites from DNA. APE1 makes an incision of the phosphodiester bond on the $5^{\prime}$-side of some damaged nucleotides and possesses $3^{\prime}-5^{\prime}$ exonuclease, 3'-phosphodiesterase, 3'phosphatase, and RNase $H$ activities. It remains unclear how such structurally different substrates can be recognized by the only active site of the enzyme. In the present work the mechanism of APE1 catalyzed target nucleotide cleavage was studied. As model substrates we used DNA- and RNAoligonucleotides forming non-canonical structures such as quadruplexes, loops and hairpins. Obtained data demonstrate the ability of APE1 to excise AP-site containing DNA as well as undamaged RNA in different non-canonical structures on the basis of substrate topology.
\end{abstract}

Keywords - AP endonuclease, non-canonical structures, fluorescence

Human apurinic/apyrimidinic (AP) endonuclease APE1 catalyses the hydrolysis of phosphodiester bonds on the $5^{\prime}$ side of an AP-site and of some damaged nucleotides such as etheno-derivatives of DNA bases [1,2], bulky photoproducts [3], benzene-derived DNA adducts [4], $\alpha$-anomers of 2' deoxynucleosides [5], oxidatively damaged pyrimidines [6] and $2^{\prime}$-deoxyuridine [7]. APE1 also possesses other activities, such as $3^{\prime}-5^{\prime}$ exonuclease [8,9], 3'-phosphodiesterase, $3^{\prime}$ phosphatase [10], and RNase H [11]. In spite of numerous studies on APE1, the molecular origin of its broad substrate specificity and the mechanism of discrimination between modified bases and/or nucleotides as well as undamaged DNA and RNA bases are not yet clear. Despite the successful characterization of crystal structures of human APE1 bound to a DNA substrate containing an F-site (a stable analogue of a natural AP-site, lacking the hydroxyl group on the $\mathrm{C} 1$-atom of ribose) or a cleaved DNA product, at present, there is no published structure of an APE1 complex with other DNA or RNA substrates.

The substrate specificity to different bases may be associated with different distortions of local DNA or RNA structure around the target base, influencing the efficiency of forming the catalytically active conformation. Another basis for substrate recognition may be related to differences in the efficiency of target base eversion from substrate into the active site of enzyme, which probably is dependent on the nature of the base and/or on stability of the target base pair in DNA or RNA.

\author{
Alexandra A. Kuznetsova \\ Institute of Chemical Biology and Fundamental Medicine \\ Siberian Branch of Russian Academy of Sciences \\ Novosibirsk, Russia \\ sandra-k@niboch.nsc.ru \\ Nikita A. Kuznetsov \\ Institute of Chemical Biology and Fundamental Medicine \\ Siberian Branch of Russian Academy of Sciences \\ Novosibirsk, Russia \\ Nikita.Kuznetsov@niboch.nsc.ru
}

The main purpose of our study was to elucidate the key steps of the mechanism behind protein-substrate interaction that ensure specific recognition of target nucleotide in various DNA- and RNA-substrates forming non-canonical structures such as quadruplexes, and various loop- and hairpincontaining structures, which can facilitate nucleotide eversion stage. The structures of these substrates were proved by CDspectroscopy. Analysis of product accumulation using gelelectrophoresis has shown that APE1 excises all of the DNA substrates used, i.e. DNA quadruplexes, containing the single F-site in core or loop regions, bulged DNA duplexes, containing the F-site, as well as undamaged RNA hairpinstructures. Obtained data demonstrate the ability of APE1 to excise AP-site containing DNA as well as undamaged RNA in different non-canonical structures. Taken together, our data support the idea that the mechanism of substrate specificity of APE1 is based on the ability of the target nucleotide to flip out of the DNA duplex into the active site owing to an enzymeinduced substrate distortion and bending.

\section{ACKNOWLEDGMENT}

This work was supported by the Russian Science Foundation No. 19-74-10034.

\section{REFERENCES}

[1] P. Prorok, C. Saint-Pierre, D. Gasparutto, O.S. Fedorova, A.A. Ishchenko, H. Leh, M. Buckle, B. Tudek, M. Saparbaev, Highly mutagenic exocyclic DNA adducts are substrates for the human nucleotide incision repair pathway, PLoS One. 7 (2012) e51776. doi:10.1371/journal.pone.0051776.

[2] P.P. Christov, S. Banerjee, M.P. Stone, C.J. Rizzo, Selective Incision of the alpha-N-Methyl-Formamidopyrimidine Anomer by Escherichia coli Endonuclease IV, J Nucleic Acids. 2010 (2010) 850234. doi: $10.4061 / 2010 / 850234$.

[3] M.G. Vrouwe, A. Pines, R.M. Overmeer, K. Hanada, L.H. Mullenders, UV-induced photolesions elicit ATR-kinase-dependent signaling in non-cycling cells through nucleotide excision repair-dependent and independent pathways, J Cell Sci. 124 (2011) 435-446. doi:10.1242/jcs.075325.

[4] A.B. Guliaev, B. Hang, B. Singer, Structural insights by molecular dynamics simulations into specificity of the major human AP endonuclease toward the benzene-derived DNA adduct, pBQ-C, Nucleic Acids Res. 32 (2004) 2844-2852. doi:10.1093/nar/gkh594.

[5] L. Gros, A.A. Ishchenko, H. Ide, R.H. Elder, M.K. Saparbaev, The major human AP endonuclease (Ape1) is involved in the nucleotide incision repair pathway, Nucleic Acids Res. 32 (2004) 73-81. doi:10.1093/nar/gkh165.

[6] S. Daviet, S. Couve-Privat, L. Gros, K. Shinozuka, H. Ide, M. Saparbaev, A.A. Ishchenko, Major oxidative products of cytosine are substrates for the nucleotide incision repair pathway, DNA Repair. 6 (2007) 8-18. doi:10.1016/j.dnarep.2006.08.001. 
[7] P. Prorok, D. Alili, C. Saint-Pierre, D. Gasparutto, D.O. Zharkov, A.A. Ishchenko, B. Tudek, M.K. Saparbaev, Uracil in duplex DNA is a substrate for the nucleotide incision repair pathway in human cells, Proc. Natl. Acad. Sci. U S A. 110 (2013) E3695-E3703. doi:10.1073/pnas.1305624110.

[8] K.-M. Chou, Y.-C. Cheng, The exonuclease activity of human apurinic/apyrimidinic endonuclease (APE1). Biochemical properties and inhibition by the natural dinucleotide Gp4G., J. Biol. Chem. 278 (2003) 18289-96. doi:10.1074/jbc.M212143200.
[9] A. Kuznetsova, O. Fedorova, N. Kuznetsov, Kinetic Features of 3'-5' Exonuclease Activity of Human AP-Endonuclease APE1, Molecules. 23 (2018) 2101. doi:10.3390/molecules23092101.

[10] D.S. Chen, T. Herman, B. Demple, Two distinct human DNA diesterases that hydrolyze 3'-blocking deoxyribose fragments from oxidized DNA, Nucleic Acids Res. 19 (1991) 5907-5914. http://www.ncbi.nlm.nih.gov/pubmed/1719484.

[11] G. Barzilay, I.D. Hickson, Structure and Function of Apurinic/Apyrimidinic Endonucleases, Bioessays. 17 (1995) 713-719. doi:DOI 10.1002/bies.950170808. 